\title{
Ajuste preciso do nível de energia na dieta de frangos de corte para controle do desempenho e da composição lipídica da carne
}

\author{
Accurate adjustment of energy level in broiler chickens diet for controlling the \\ performance and the lipid composition of meat
}

\author{
Gabriela da Silva Ferreira ${ }^{\mathrm{I}^{*}}$ Marcos Franke Pinto ${ }^{\mathrm{II}}$ Manoel Garcia Neto ${ }^{\mathrm{II}}$

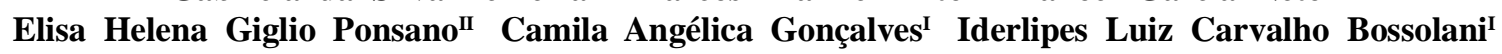 \\ Amanda Gomes Pereira ${ }^{\mathrm{I}}$
}

\section{RESUMO}

O objetivo deste experimento foi avaliar a influência da variação do nível de energia metabolizável na dieta de frangos de corte, associada a uma variação proporcional da densidade nutricional da ração, sobre o desempenho das aves e sobre a composição da fração lipídica da carne. Foram avaliados separadamente machos e fêmeas da linhagem Cobb 500. Para análise de desempenho, foi utilizado um delineamento inteiramente casualizado em esquema fatorial $6 \times 3$, sendo seis níveis de energia metabolizável na ração $(2.800,2.900,3.000$, $3.100,3.200$ e $\left.3.300 \mathrm{kcal} \mathrm{kg}^{-1}\right)$ e três idades ao abate $(42,49$ e 56 dias). Foi utilizada a metodologia de superfície de resposta para estabelecer um modelo matemático, explicando o comportamento do peso vivo, do consumo de ração e da conversão alimentar. Para a avaliação da composição da fração lipídica da carne, determinou-se o teor de lipídeos totais e de colesterol na carne do peito, na coxa com pele e na coxa sem pele, seguindo um delineamento inteiramente casualizado em esquema fatorial $3 \times 3 \times 2$, sendo três níveis de energia metabolizável na ração $\left(2.800,3.000\right.$ e $3.300 \mathrm{kcal} \mathrm{kg}^{-1}$ de ração), três idades ao abate (42, 49 e 56 dias) e dois sexos. A redução da energia metabolizável da ração até valores próximos de $3.000 \mathrm{kcal}^{\mathrm{kg}^{-1}}$ não afetou o peso vivo, mas, reduzindo a energia da ração abaixo desse valor, o peso vivo diminui. $O$ consumo de ração foi menor quando o nível energético da ração foi mais alto. A conversão alimentar melhorou de forma proporcional ao aumento do nível energético da ração. O desempenho de todas as aves ficou dentro da faixa considerada adequada para a linhagem. A carne de peito apresentou menos lipídeos totais e colesterol que a carne da coxa. O teor de lipídeos totais da coxa com pele foi mais que o dobro da coxa sem pele, mas o teor de colesterol não diferiu com a retirada da pele, demonstrando que o colesterol não está associado à gordura subcutânea. O teor de gordura intramuscular foi menor na carne das aves que receberam a ração com menor nível energético. Essas informações podem auxiliar na definição do manejo nutricional mais adequado. Apesar da redução no desempenho produtivo, a restrição da energia na ração dos frangos pode ser uma alternativa viável, se os consumidores estiverem dispostos a pagar mais por carne com menos gordura.

Palavras-chave: frango de corte, carne, qualidade, lipídios, alimentos, teor de colesterol.

\section{ABSTRACT}

The purpose of this experiment was to investigate the influence of changes in dietary metabolizable energy level, associated with a proportional nutrient density variation, on broiler chickens performance and on the lipid composition of meat. Males and females Cobb 500 broilers were evaluated separately. Performance evaluation followed a completely randomized design with factorial $6 \times 3$ arrangement - six energy levels $\left(2,800,2,900,3,000,3,100,3,200\right.$ and 3,300 $\left.\mathrm{kcal} \mathrm{kg}^{-1}\right)$ and three slaughter ages (42, 49 and 56 days). Response surface methodology was used to establish a mathematical model to explain live weight, feed intake and feed conversion behavior. Total lipids and cholesterol were determined in breast meat and in thigh meat, with and without skin. For lipid composition analysis, a $3 \times 3 \times 2$ factorial arrangement in a completely randomized design - three ration's metabolizable energy levels $(2,800,3,000$ and 3,300 $\mathrm{ccal}^{\mathrm{kg}^{-1}}$ ), three slaughter ages (42, 49 and 56 days) and two sexes - was used. The reduction in the diet metabolizable energy close to $3,000 \mathrm{kcal}^{\mathrm{kg}-1}$ did not affect live weight, but below this value, live weight decreased. Feed intake was lower when the dietary energy level was higher. Feed conversion was favored in a direct proportion to the increase of the energy level of the diet. The performance of all birds was within the range considered appropriate for the lineage. Breast meat had less total lipids and cholesterol than thigh meat. Thigh with skin had more than the double of the amount of total lipids than skinless thigh, but the cholesterol content did not differ with the removal of the skin. Intramuscular fat content was lower in the meat from birds fed with lower energy level ration. This information may help

'Programa de Pós-graduação em Ciência Animal, Faculdade de Medicina Veterinária de Araçatuba (FMVA), Universidade Estadual Paulista Júlio de Mesquita Filho (UNESP), 16050-680, Araçatuba, SP, Brasil. E-mail: gabrielasf2@ @otmail.com. *Autor para correspondência.

"Área de Medicina Veterinária Preventiva e Produção Animal, FMVA, UNESP, Araçatuba, SP, Brasil. 
to define the most appropriate nutritional management. Despite the decrease in bird's productive performance, the restriction of energy in broiler chickens feed may be a viable alternative, if the consumers are willing to pay more for meat with less fat.

Key words: broiler chicken, meat, quality, lipids, food, cholesterol content.

\section{INTRODUÇÃO}

O Brasil ocupa hoje uma posição consolidada entre os maiores produtores mundiais de carne de frango, sendo, desde 2004, o maior exportador mundial do produto (UBABEF, 2012). A manutenção desse bom desempenho, num mercado altamente competitivo, exige uma evolução constante, enfocando não apenas os aspectos econômicos e de produtividade, mas também aqueles ligados à qualidade e atendimento das demandas dos consumidores.

Cada vez mais, o teor de gordura da carne vem sendo considerado um fator determinante para sua qualidade (NASCIF et al., 2004). Segundo MOURA (2003), a evolução do conhecimento científico, assim como a influência da mídia sobre o comportamento da sociedade moderna, tem contribuído para uma maior conscientização dos consumidores, que passaram a demonstrar maior preocupação com a saúde. Tal fato se traduz na procura mais acentuada por alimentos saudáveis, com baixos níveis de gorduras, principalmente as saturadas, e colesterol. A produção de carne com menor teor de gordura é vantajosa também no aspecto econômico, uma vez que a síntese de gordura requer mais energia que a do tecido muscular, sendo, portanto, mais dispendiosa.

A redução do teor de gordura na carne de frangos pode ser alcançada pelo melhoramento genético e pela adequação dos níveis de energia e demais nutrientes da dieta (SOUZA, 2010). De acordo com MENDES et al. (2004), o nível energético da dieta influencia diretamente o desempenho de frangos de corte. O aumento do nível de energia das rações proporciona maior ganho de peso e melhor conversão alimentar, porém acarreta aumento na deposição de gordura (WATANABE et al., 2001). Toda energia que ultrapassa as exigências da ave para mantença e deposição de tecido muscular é armazenada como gordura (KESSLER et al., 2000).

Nesse quadro, a precisão na formulação da ração torna-se fundamental. Deve ser considerado não apenas o teor de proteína bruta, mas a digestibilidade dos aminoácidos; não apenas a quantidade dos minerais, mas a sua disponibilidade. Isso promove uma utilização mais eficiente da energia da dieta, o que, além de beneficiar o aspecto econômico, reduz o desperdício de nutrientes nas excretas e, consequentemente, o impacto ambiental (PENZJUNIOR et al., 2009).

Desta forma, o presente trabalho teve como objetivo avaliar a influência do nível energético na dieta de frangos de corte em diferentes idades de abate sobre o desempenho das aves e sobre a composição da fração lipídica da carne.

\section{MATERIAL E MÉTODOS}

Para análise de desempenho, foram avaliados separadamente 240 frangos machos e 240 fêmeas da linhagem Cobb 500. Foi utilizado um delineamento inteiramente casualizado em esquema fatorial $6 \times 3$, sendo seis níveis de energia metabolizável $(2.800,2.900,3.000,3.100,3.200 \mathrm{e}$ $\left.3.300 \mathrm{kcal} \mathrm{kg}^{-1}\right)$ e três idades ao abate $(42,49$ e 56 dias), com quatro repetições por tratamento. Foi utilizada a metodologia de superfície de resposta para estabelecer um modelo matemático, explicando o comportamento do peso vivo, do consumo de ração e da conversão alimentar (RODRIGUES \& IEMMA, 2009), segundo os procedimentos do PROC RSREG do programa estatístico SAS (2008).

As aves foram alojadas em galpão de alvenaria, dispostas em boxes com dez aves cada, com bebedouros e comedouros automáticos e cama de maravalha. Os pintinhos, com um dia de idade, foram pesados e distribuídos ao acaso nos boxes. As dietas experimentais, distintas para machos e fêmeas, foram formuladas com a utilização do Programa Prático de Formulação de Rações (PPFR) (GARCIA NETO, 2013). A composição das dietas seguiu as recomendações de ROSTAGNO et al. (2005), mantendo a relação entre energia e os demais nutrientes, o que resultou em rações com diferentes densidades nutricionais (Tabela 1). O oferecimento de ração e água foi ad libitum.

Aos 42, 49 e 56 dias de idade, todas as aves e a ração de cada boxe foram pesadas para avaliação de desempenho. Uma ave de cada boxe, com o peso próximo à média do lote $(10 \%$ para mais ou para menos) foi abatida no abatedouro experimental da Faculdade, após jejum alimentar de seis horas, segundo as exigências legais (BRASIL, 1998).

Para análise da composição da fração lipídica da carne, foi empregado um delineamento fatorial de $3 \times 3 \times 2$, sendo três níveis de energia metabolizável da ração $\left(2.800,3.000\right.$ e $\left.3.300 \mathrm{kcalkg}^{-1}\right)$, três idades ao abate (42, 49 e 56 dias) e dois sexos, com três repetições por tratamento. Foram tomadas 
Tabela 1 - Composição percentual das rações experimentais, segundo as fases e as exigências dos machos e fêmeas.

\begin{tabular}{|c|c|c|c|c|c|c|c|c|c|c|c|c|}
\hline \multirow{2}{*}{$\begin{array}{l}\text { Ingredientes } \\
\text { Milho }\end{array}$} & \multicolumn{2}{|c|}{$\begin{array}{c}\text { Inicial } \\
\text { (1-21 dias) }\end{array}$} & \multicolumn{2}{|c|}{$\begin{array}{l}\text { Crescimento } \\
\text { (22-35 dias) }\end{array}$} & \multicolumn{2}{|c|}{$\begin{array}{r}\text { Terminação } \\
\text { (36-58 dias) }\end{array}$} & \multicolumn{2}{|c|}{$\begin{array}{c}\text { Inicial } \\
\text { (1-21 dias) }\end{array}$} & \multicolumn{2}{|c|}{$\begin{array}{r}\text { Crescimento } \\
\text { (22-35 dias) }\end{array}$} & \multicolumn{2}{|c|}{$\begin{array}{l}\text { Terminação } \\
\text { (36-58 dias) }\end{array}$} \\
\hline & 60,79 & 41,89 & 64,65 & 52,25 & 69,24 & 64,51 & 60,93 & 42,64 & 66,92 & 57,71 & 70,53 & 67,43 \\
\hline Óleo de soja & 0,00 & 10,09 & 0,00 & 8,12 & 0,00 & 5,80 & 0,00 & 9,89 & 0,00 & 7,14 & 0,00 & 5,29 \\
\hline Farelo de soja $-45 \%$ & 32,20 & 43,35 & 26,56 & 35,65 & 19,74 & 26,36 & 32,01 & 43,01 & 23,22 & 31,36 & 18,15 & 24,34 \\
\hline Fosfato bicálcico & 1,71 & 2,10 & 1,39 & 1,70 & 1,15 & 1,40 & 1,61 & 1,97 & 1,32 & 1,61 & 1,07 & 1,31 \\
\hline Sal comum & 0,47 & 0,56 & 0,41 & 0,49 & 0,36 & 0,43 & 0,44 & 0,53 & 0,39 & 0,46 & 0,34 & 0,41 \\
\hline L-lisina $\mathrm{HCl}$ & 0,22 & 0,16 & 0,20 & 0,15 & 0,22 & 0,20 & 0,22 & 0,16 & 0,20 & 0,15 & 0,10 & 0,06 \\
\hline DL-metionina & 0,24 & 0,32 & 0,19 & 0,25 & 0,14 & 0,19 & 0,24 & 0,32 & 0,16 & 0,21 & 0,06 & 0,09 \\
\hline $\mathrm{L}$ treonina & 0,05 & 0,06 & 0,03 & 0,04 & 0,03 & 0,03 & 0,05 & 0,06 & 0,03 & 0,03 & 0,00 & 0,00 \\
\hline Calcário calcítico & 0,87 & 0,96 & 0,76 & 0,85 & 0,68 & 0,76 & 0,82 & 0,91 & 0,73 & 0,82 & 0,65 & 0,73 \\
\hline Inerte (caulim) & 2,22 & 0,00 & 5,30 & 0,00 & 8,12 & 0,00 & 3,17 & 0,00 & 6,53 & 0,00 & 8,76 & 0,00 \\
\hline Premix min. e vit.* & 0,52 & 0,52 & 0,51 & 0,51 & 0,33 & 0,33 & 0,52 & 0,52 & 0,51 & 0,51 & 0,33 & 0,33 \\
\hline Energ. met. $\left(\mathrm{kcal} \mathrm{kg}^{-1}\right) * *$ & 2.800 & 3.300 & 2.800 & 3.300 & 2.800 & 3.300 & 2.800 & 3.300 & 2.800 & 3.300 & 2.800 & 3.300 \\
\hline Proteína bruta (\%) & 20,00 & 23,48 & 17,70 & 20,79 & 14,97 & 17,59 & 19,92 & 23,39 & 16,35 & 19,27 & 14,18 & 16,72 \\
\hline Cálcio (\%) & 0,85 & 1,00 & 0,72 & 0,84 & 0,61 & 0,72 & 0,80 & 0,95 & 0,68 & 0,80 & 0,58 & 0,68 \\
\hline P disponível (\%) & 0,42 & 0,50 & 0,36 & 0,42 & 0,30 & 0,36 & 0,40 & 0,48 & 0,34 & 0,40 & 0,29 & 0,34 \\
\hline Potássio (\%) & 0,76 & 0,91 & 0,67 & 0,80 & 0,56 & 0,66 & 0,76 & 0,91 & 0,61 & 0,74 & 0,53 & 0,63 \\
\hline Sódio $(\%)$ & 0,20 & 0,24 & 0,18 & 0,21 & 0,16 & 0,19 & 0,19 & 0,23 & 0,17 & 0,20 & 0,15 & 0,18 \\
\hline Cloro $(\%)$ & 0,37 & 0,41 & 0,33 & 0,36 & 0,30 & 0,34 & 0,35 & 0,39 & 0,31 & 0,35 & 0,27 & 0,30 \\
\hline Lisina dig. $(\%)$ & 1,11 & 1,31 & 0,96 & 1,13 & 0,81 & 0,95 & 1,11 & 1,31 & 0,88 & 1,04 & 0,69 & 0,81 \\
\hline Metionina dig. (\%) & 0,52 & 0,63 & 0,44 & 0,54 & 0,36 & 0,44 & 0,51 & 0,63 & 0,40 & 0,48 & 0,28 & 0,34 \\
\hline Met. + cistina dig. $(\%)$ & 0,79 & 0,93 & 0,69 & 0,81 & 0,58 & 0,69 & 0,79 & 0,93 & 0,63 & 0,75 & 0,50 & 0,58 \\
\hline Triptofano dig. (\%) & 0,22 & 0,27 & 0,19 & 0,23 & 0,15 & 0,19 & 0,22 & 0,27 & 0,17 & 0,21 & 0,14 & 0,18 \\
\hline Treonina dig. (\%) & 0,72 & 0,85 & 0,62 & 0,74 & 0,53 & 0,62 & 0,72 & 0,85 & 0,57 & 0,67 & 0,48 & 0,56 \\
\hline Relação Energia:Prot. & 140,0 & 140,5 & 158,2 & 158,8 & 187,0 & 187,6 & 140,5 & 141,1 & 171,3 & 171,3 & 197,4 & 197,4 \\
\hline
\end{tabular}

*Suplementos vitamínico-minerais utilizados nas rações em três fases de criação (quantidade/kg do produto) incluíram: INICIAL: vit. A 1.670.000UI; vit. D3 - 335.000 UI; vit. E - 2.500mg; vit. K3 - 417mg; vit. B1 - 250mg; vit. B2 - 835 mg; vit. B6 - $250 \mathrm{mg}$; vit. B12 2.000mcg; ácido fólico - 100mg; biotina - 9mg; niacina - 5.835mg; pantotenato de cálcio - 1.870mg; Cu - 1.000mg; Co - $17 \mathrm{mg}$; I - 170mg; Fe - 8.335mg; Mn - 10.835mg; Zn - 7.500mg; Se - 35 mg; cloreto de colina 50\% - 116.670mg; metionina - 250.000mg; coccidiostático 13.335mg; promotor de crescimento - 13.335mg; antioxidante - 2.000mg. CRESCIMENTO: vit. A - 1.335.000 UI; vit. D3 - 300.000UI; vit. E - 2.000mg; vit. K3 - 335mg; vit. B1 - 167mg; vit. B2 - 670mg; vit. B6 - 170mg; vit. B12 - 1.670mcg; ácido fólico - 67mg; biotina - 7mg; niacina - 4.670mg; pantotenato de cálcio - 1.870mg; Cu - 1.000mg; Co - 17mg; I - 170mg; Fe - 8.335mg; Mn - 10.835mg; Zn - 7.500mg; Se - 35mg; cloreto de colina 50\% - 83.340mg; metionina - 235.000mg; coccidiostático - $10.000 \mathrm{mg}$; promotor de crescimento - $10.000 \mathrm{mg}$; antioxidante - 2.000mg. TERMINAÇÃO: vit. A - 1.670.000UI; vit. D3 - 335.000UI; vit. E - 2.335mg; vit. K3 - 400mg; vit. B1 - 100mg; vit. B2 - 800mg; vit. B6 - 200mg; vit. B12 - 2.000mcg; ácido fólico - 67mg; biotina - 7mg; niacina - 5.670mg; pantotenato de cálcio - 2.000mg; $\mathrm{Cu}-2.000 \mathrm{mg}$; Co - 27mg; I - 270mg; Fe - 16.670mg; Mn - 17.335mg; Zn - 12.000mg; Se - 70mg; cloreto de colina 50\% - 100.000mg; metionina - 235.000mg; antioxidante - 2.000mg.

**A composição das rações com valores intermediários de energia metabolizável (2.900, 3.000, 3.100 e 3.200 kcal.kg $\left.{ }^{-1}\right)$ não é apresentada.

amostras de coxa com pele, coxa sem pele e peito sem pele, por serem as formas mais comuns de consumo. As amostras foram desossadas, moídas e homogeneizadas em processador de alimentos. Em seguida, foram congeladas, liofilizadas e armazenadas sob congelamento, para posterior determinação do teor de lipídeos totais e de colesterol. O teor de lipídeos totais foi determinado gravimetricamente após extração em aparelho de Soxhlet, utilizando éter como solvente, e o teor de colesterol foi determinado espectrofotometricamente a $625 \mathrm{~nm}$, após extração com clorofórmio e reação colorimétrica, conforme descrito por INSTITUTO ADOLFO LUTZ (2008).
Os resultados das análises foram submetidos ao teste t com 5\% de significância (ZAR, 2009), segundo o procedimento PROC GLM do programa estatístico SAS (2008).

\section{RESULTADOS E DISCUSSÃO}

Desempenho das aves

Os modelos matemáticos foram altamente significativos para as variáveis estudadas, demonstrando um bom ajuste aos dados experimentais, com alta porcentagem de variação explicada $\left(\mathrm{R}^{2}\right)$ (SAMPAIO, 2007). Com base nos 
modelos matemáticos foram construídos os gráficos apresentados na figura 1 . Nos gráficos A e B é possível observar que, tanto para machos como para fêmeas, a diminuição do nível energético e da densidade nutricional da ração não causou diminuição do peso

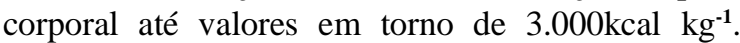
Observação semelhante foi relatada por MENDES et al. (2004), que, avaliando diferentes níveis energéticos na ração de frangos dos 21 aos 42 dias de idade, não observaram diferenças significativas para peso vivo. Porém, quando a energia metabolizável da ração se aproxima do limite inferior avaliado neste trabalho, começa a haver prejuízo do peso corporal. Mas é importante destacar que todas as aves deste experimento, mesmo aquelas que apresentaram peso corporal mais baixo, alcançaram a meta de desempenho previsto no Manual de Manejo da linhagem Cobb 500 (COBB VANTRESS, 2001).

A influência do nível energético e da densidade nutricional da ração sobre a conversão

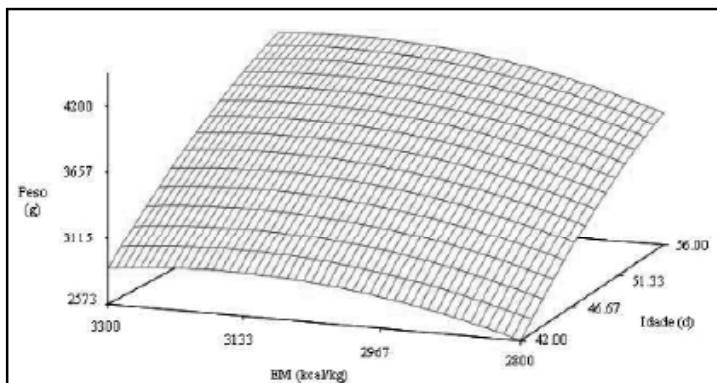

(A) Peso vivo - machos

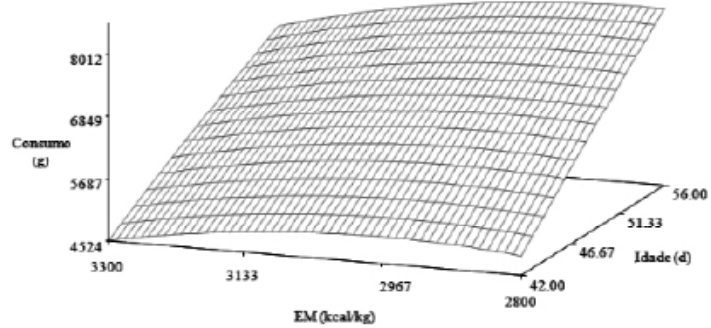

(C) Consumo de ração - machos

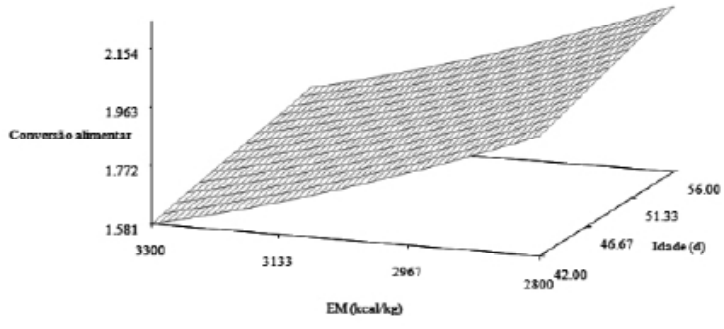

(E) Conversão alimentar - machos

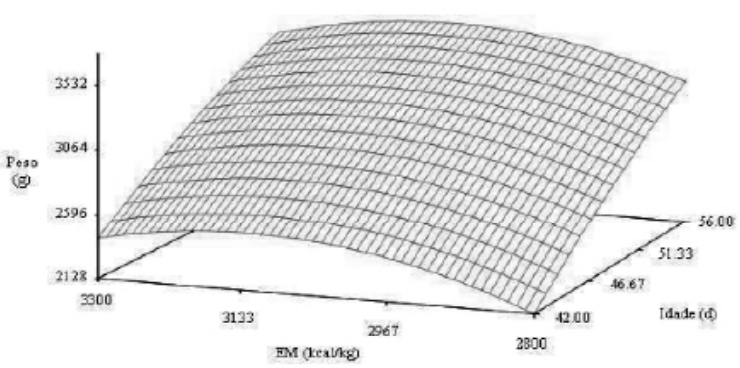

(B) Peso vivo - fêmeas

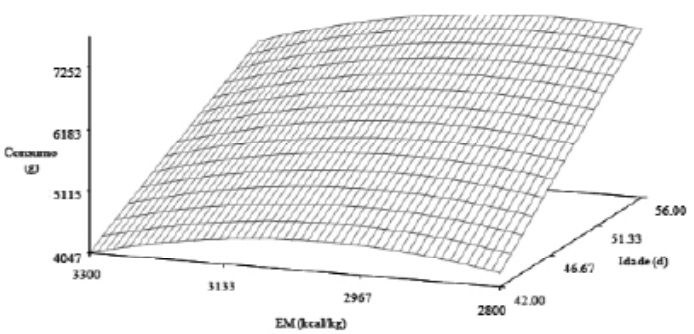

(D) Consumo de ração - fêmeas

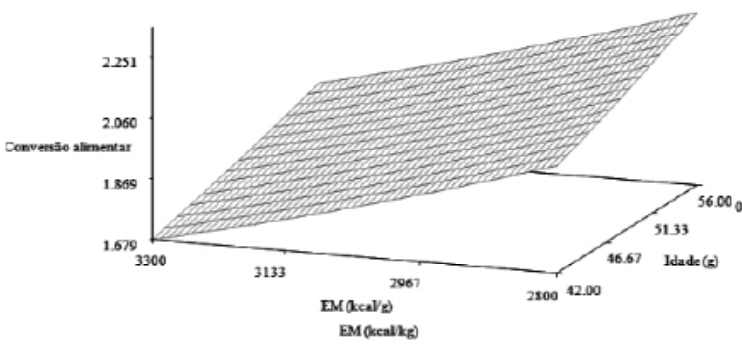

(F) Conversão alimentar - fêmeas

Figura 1 - Superfícies de resposta para os parâmetros de desempenho produtivo (peso vivo, consumo de ração e conversão alimentar) dos machos e fêmeas, em função da idade e do nível de energia metabolizável (EM) da ração. 
alimentar das aves de ambos os sexos é bastante clara, conforme pode ser observado nos gráficos $\mathrm{E}$ e $\mathrm{F}$ da figura 1. A conversão alimentar é favorecida pelo aumento do nível energético da ração, como consequência do aumento de ganho de peso e da diminuição do consumo de ração. O modelo matemático revelou que, para a conversão alimentar, apenas a regressão linear foi significativa pelo teste de Fisher $(\mathrm{P}>\mathrm{F})$, demonstrando que essa influência segue um comportamento linear.

Ainda comparando os gráficos de consumo de ração (Figura 1, C e D) e de peso corporal (Figura 1, A e B), observa-se que o consumo diminui em função da diminuição do peso corporal das aves, e essa tendência é observada em ambos os sexos. Se for levado em consideração que a energia representa, em geral, o nutriente mais caro da ração e que, conforme já foi apresentado, mesmo as aves com menor peso corporal apresentaram desempenho dentro dos valores considerados adequados para a linhagem (COBB VANTRESS, 2001), a decisão sobre qual manejo nutricional deve ser adotado deve ser muito bem analisada, levando em consideração o preço do frango e o custo da ração.

\section{Composição da fração lipídica da carne}

O teor de lipídeos totais e de colesterol da carne não sofreu influência do sexo ou da idade das aves em nenhum dos cortes analisados. Por esse motivo, na tabela 2, são apresentadas as médias dessas variáveis apenas em função do nível de energia da ração. O teor de lipídeos totais variou entre os cortes estudados, tendo sido observada menor porcentagem de lipídeos totais e de colesterol na carne do peito $(\mathrm{P}<0,05)$. $\mathrm{O}$ teor de lipídeos totais da coxa com pele foi mais que o dobro do valor observado na coxa sem pele, demonstrando que uma parcela relevante da gordura dos cortes encontra-se depositada no tecido subcutâneo (AJUYAH et al., 1991). Por outro lado, não foi observada diferença significativa entre os teores de colesterol da coxa com pele e sem pele $(\mathrm{P}>0,05)$, demonstrando que esse constituinte está presente no tecido muscular e não na gordura subcutânea. A Tabela Brasileira de Composição de Alimentos (TACO, 2011) e as tabelas de composição de alimentos publicadas por PHILIPPI (2002) e USDA (2001) também demonstram que o teor de colesterol da coxa de frango com pele e sem pele é semelhante. NOVELLO et al. (2006) também observaram que o conteúdo de colesterol das carnes não está claramente relacionado ao conteúdo de gordura.

As aves que receberam menor nível energético na dieta apresentaram menor teor de lipídeos na coxa sem pele e no peito $(\mathrm{P}<0,05)$, demonstrando a influência da densidade nutricional da ração na deposição de gordura intramuscular. Essa influência não é observada na coxa com pele, pois a presença de grande quantidade de gordura subcutânea aumenta o coeficiente de variação e mascara a diferença entre as médias de lipídeos totais das amostras. A deposição de gordura subcutânea e intramuscular é bastante estudada em bovinos, por ser um fator determinante para a textura e palatabilidade da carne (JEREMIAH, 1996). Em frangos de corte, esse aspecto não tem sido tão explorado, talvez porque a maciez não seja um problema tão importante na carne de frango como na carne bovina. Mas sabese que esse padrão de deposição é influenciado por fatores intrínsecos - como raça - e extrínsecos - entre os quais a energia da dieta dos animais tem uma importância fundamental.

As médias encontradas neste trabalho, tanto para lipídeos totais como para colesterol, foram superiores aos valores reportados em outras publicações, como a Tabela Brasileira de Composição de Alimentos (TACO, 2011), e as tabelas de PHILIPPI (2002) e USDA (2001). No entanto, a relação entre os constituintes presentes na fração lipídica dos cortes - lipídeos totais e colesterol - é semelhante. No presente trabalho, as amostras de carne foram

Tabela 2 - Análise de colesterol (mg/g) e lipídeos totais (\%) da carne de frangos de corte (base seca), em função dos cortes de carne e do nível de energia metabolizável (EM) da ração.

\begin{tabular}{|c|c|c|c|c|c|c|}
\hline EM (kcal) & Coxa com pele & Coxa sem pele & Peito & Coxa com pele & Coxa sem pele & Peito \\
\hline 2800 & $2,13^{\mathrm{A}} \pm 0,51$ & $2,21^{\mathrm{A}} \pm 0,60$ & $1,69^{\mathrm{B}} \pm 0,51$ & $33,44^{\mathrm{A}} \pm 10,26$ & $12,79^{\mathrm{Bb}} \pm 2,79$ & $5,54^{\mathrm{Cb}} \pm 1,55$ \\
\hline 3000 & $2,11^{\mathrm{A}} \pm 0,53$ & $2,34^{\mathrm{A}} \pm 0,62$ & $1,65^{\mathrm{B}} \pm 0,44$ & $35,02^{\mathrm{A}} \pm 8,66$ & $15,05^{\mathrm{Bab}} \pm 3,66$ & $7,38^{\mathrm{Ca}} \pm 2,15$ \\
\hline 3300 & $2,39^{\mathrm{A}} \pm 0,54$ & $2,32^{\mathrm{A}} \pm 0,64$ & $1,68^{\mathrm{B}} \pm 0,54$ & $34,26^{\mathrm{A}} \pm 11,33$ & $15,83^{\mathrm{Ba}} \pm 4,28$ & $6,92^{\mathrm{Ca}} \pm 2,22$ \\
\hline
\end{tabular}

Médias seguidas de letras distintas, maiúsculas nas linhas e minúsculas nas colunas diferem entre si pelo teste $\mathrm{t}(\mathrm{P}<0,05)$. 
moídas, liofilizadas e trituradas antes de serem submetidas à extração de lipídeos. Essa modificação na técnica de determinação de lipídeos tem sido utilizada em nosso laboratório a partir do trabalho de ALMEIDA (2007), quando foi observada a dificuldade para a penetração do solvente na carne moída in natura. Essa modificação da técnica é, certamente, a razão principal da diferença entre os resultados deste trabalho e os resultados apresentados em outras publicações. Esta é uma observação muito importante, pois sugere que os resultados da análise de lipídeos obtidos com a carne em seu estado fresco podem ser subestimados. Além disso, as aves foram criadas em galpão climatizado, com poucas aves por grupo e, consequentemente, sem dificuldade para acesso aos comedouros e bebedouros, e toda essa situação de conforto certamente beneficiou a deposição de gordura.

Assim, com a realização deste trabalho, foi possível demonstrar que a restrição da energia na dieta das aves pode diminuir o teor de gordura intramuscular. Não foi possível demonstrar diretamente a possibilidade de reduzir o teor de colesterol da carne de frango pela diminuição do nível energético da ração, mas isso pode ser atribuído à técnica de determinação de colesterol, que é uma técnica de difícil padronização, caracterizada por uma grande variabilidade e um alto coeficiente de variação, o que diminui a consistência dos resultados.

\section{CONCLUSÃO}

Com a restrição do teor energético da dieta de frangos de corte, associada ao ajuste preciso da densidade nutricional da ração, é possível produzir carne de frango com menor teor de gordura intramuscular e, portanto, mais saudável aos consumidores.

Aconversão alimentar das aves é prejudicada, de forma linear, pela diminuição do nível energético da ração, em todas as idades de abate estudadas.

Essa alternativa de manejo nutricional pode ser interessante para atender a demanda dos consumidores dispostos a pagar mais por carne com menor teor de gordura, abrindo uma perspectiva de diversificação dos produtos avícolas.

\section{REFERÊNCIAS}

AJUYAH, A.O. et al. Changes in the fatty acid composition of whole carcass and selected meat portions of broiler chicks fed full-fat oil seeds. Poultry Science, v.70, p.2304-2314, 1991. Disponível em: <http://ps.oxfordjournals.org/content/70/11/2304. full.pdf + html? sid=f6ba 19ae-b11d-42fd-89f9-8ae226569e69>. Acesso em: 12 març. 2012. doi: 10.3382/ps.0702304

ALMEIDA, A.P.S. Efeito do óleo de linhaça no desempenho, características de carcaça e qualidade da carne de frangos de corte. 2007. 99f. Dissertação (Mestrado em Ciência Animal) - Faculdade de Odontologia, Universidade Estadual Paulista, Araçatuba, SP.

BRASIL. Ministério da Agricultura, Pecuária e Abastecimento. Portaria n.210, de 10 de novembro de 1998. Aprova o Regulamento Técnico de Inspeção Tecnológica e Higiênico-Sanitária de Carne de Aves. Diário Oficial da República Federativa do Brasil, Brasília, DF, 26 nov. 1998. Seção 1, p.226.

COBB VANTRESS. Manual de manejo de frangos Cobb 500: guia de manejo. São Paulo. Cobb Vantress Brasil, 2001. 47p.

GARCIA-NETO, M. PPFR: Programa prático para formulação de ração - versão Excel 2010. Disponível em: <https://sites.google. com/site/ppfrparaexcel2007ousuperior/>. Acesso em: 23 set. 2013.

INSTITUTO ADOLFO LUTZ. Métodos físico químicos para análise de alimentos. São Paulo, 2008. Disponível em: <http://www.ial.sp.gov.br/index.php?option=com_remository $\&$ Itemid $=7 \&$ func $=$ select $\&$ orderby $=1 \&$ Itemid $=7>$. Acesso em: 10 abr. 2011

JEREMIAH, L.E. The influence of subcutaneous fat thickness and marbling on beef - palatability and consumer aceitability. Food Research International, v.29, n.5-6, p.513-520, 1996. Disponível em: <http://www.sciencedirect.com/science/article/pii/ S096399699600049X>. Acesso em: 23 set. 2012. doi: 10.1016/ S0963-9969(96)00049-X

KESSLER, A.M. et al. Manipulação da quantidade de gordura na carcaça de frangos. In: CONFERÊNCIA APINCO 2000 DE CIÊNCIA E TECNOLOGIA AVÍCOLAS, 2000, Campinas, SP. Anais... Campinas: FACTA, 2000. p.107-133.

MENDES, A.A. et al. Efeitos da energia da dieta sobre desempenho, rendimento de carcaça e gordura abdominal de frangos de corte. Revista Brasileira de Zootecnia, v.33, n.6, suppl.3, p.2300-2307, 2004. Disponível em: <http://www.scielo.br/scielo.php?script=sci_arttext\&pid $=$ S1516-35982004000900016 $>$. Acesso em: 22 set. 2012. doi: 10.1590/S1516-35982004000900016.

MOURA, B.H.S. Desempenho e composição da carcaça de frangos de corte alimentados com diferentes níveis energéticos, com e sem óleo. 2003. 51f. Dissertação (Mestrado em Medicina Veterinária) - Universidade Federal de Minas Gerais, MG.

NASCIF, C.C.C. et al. Determinação dos valores energéticos de alguns óleos e gorduras para pintos de corte machos e fêmeas aos 21 dias de idade. Revista Brasileira de Zootecnia, v.33, n.2. p.375-385, 2004. Disponível em: <http://www.scielo.br/scielo. php ?script $=$ sci_arttext $\&$ pid $=$ S 1516-35982004000200014>. Acesso em: 22 set. 2012. doi: 10.1590/S151635982004000200014 .

NOVELLO, D. et al. Teor de gordura e colesterol na carne suína, bovina e de frango. Nutrire: Revista da Sociedade Brasileira de Alimentação e Nutrição, v.31, n.2, p.103-121, 2006. Disponível em: <http://www.revistanutrire.org.br/articles/view/ id/4f996c3a1 ef1fad40e000006>. Acesso em: 22 set. 2012. 
PENZ-JUNIOR, A.M.; BRUNO, D.; FIGUEIREDO, A. Nutrição de frangos-mudanças de paradigmas para o futuro. In: Anais da conferência FACTA 2009 de ciência e Tecnologia Avícolas. Porto Alegre, 2009. Anais... Porto Alegre, RS, 2009.

PHILIPPI, S.T. Tabela de composição de alimentos: suporte para decisão nutricional. 2.ed. São Paulo: Coronário, 2002. 107p.

RODRIGUES, M.S.; IEMMA, A.F. Planejamento de experimentos e otimização de processos. 2.ed. Campinas: Casa do Espírito Amigo Fraternidade Fé e Amor, 2009. 358p.

ROSTAGNO, H.S. et al. Tabelas brasileiras para aves e suínos: composição de alimentos e exigências nutricionais. 2.ed. Viçosa: UFV, Departamento de Zootecnia, 2005. 186p.

SAMPAIO, I.B.M. Estatística aplicada à experimentação animal. 3.ed. Belo Horizonte: Fundação de estudo e pesquisa em medicina veterinária e zootecnia, 2007. 264p.

SAS Institute Inc. SAS/STAT 9.2 User's guide. Cary, 2008. 26p.

SOUZA, L. M.G. et al. Influência do cromo no desempenho, na qualidade da carne e no teor de lipídeos no plasma sanguíneo de frangos de corte. Revista Brasileira de
Zootecnia, v.39, n.4, p.808-814, 2010. Disponível em: <http://www.scielo.br/scielo.php?script=sci_arttext\&pid =S1516-35982010000400016>. Acesso em: 12 ago 2012. doi: $10.1590 / \mathrm{S} 151635982010000400016$.

TACO. Tabela brasileira de composição de alimentos. 4.ed.rev. ampl. Campinas: NEPAUNICAMP, 2011. 161p.

UBABEF (UNIÃO BRASILEIRA DE AVICULTURA). Relatório Anual de 2012. Disponível em: <http://www.abef.com.br/ubabef/ exibenoticiaubabef.php?notcodigo=3293>. Acesso em: 03 out. 2012.

USDA. Nutrient database for standard reference: release 14 . U.S.A: Department of Agriculture: Agricultural Research Service, 2001. Disponível em: 〈http://ndb.nal.usda.gov/ndb/search/list>. Acesso em: 22 set 2012

WATANABE, K. et al. Efeito do nível de energia metabolizável da dieta sobre o metabolismo energético de frangos de corte. In: REUNIÃO ANUAL DA SOCIEDADE BRASILEIRA DE ZOOTECNIA, 38., 2001, Piracicaba, SP. Anais... Piracicaba: Sociedade Brasileira de Zootecnia, 2001. p.762.

ZAR, J.H. Biostatistical analysis. 5.ed. New Jersey: PrenticeHall, 2009. 994p. 